BNL-108540-2015-JA

\title{
Interaction of CuS and Sulfur in Li-S battery system
}

\author{
Ke Sun ${ }^{1}$, Dong Su${ }^{2}$, Qing Zhang ${ }^{3}$, David C. Bock ${ }^{1}$, Amy C. Marschilok ${ }^{3,4}$, Kenneth J. Takeuchi ${ }^{3,4}$, \\ Esther S. Takeuchi ${ }^{1,3,4}$, and Hong Gan ${ }^{12}$
}

1. Energy Sciences Directorate, Brookhaven National Laboratory, Upton, NY 11973

2. Center for Functional Nanomaterials, Brookhaven National Laboratory, Upton, NY 11973

3. Department of Materials Science and Engineering, Stony Brook University, Stony Brook, NY 11794

4. Department of Chemistry, Stony Brook University, Stony Brook, NY 11794

\begin{abstract}
Lithium-Sulfur (Li-S) battery has been a subject of intensive research in recent years due to its potential to provide much higher energy density and lower cost than the current state of the art lithiumion battery technology. In this work, we have investigated Cupric Sulfide (CUS) as a capacitycontributing conductive additive to the sulfur electrode in a Li-S battery. Galvanostatic charge/discharge cycling has been used to compare the performance of both sulfur electrodes and S:CuS hybrid electrodes with various ratios. We found that the conductive CuS additive enhanced the utilization of the sulfur cathode under a $1 \mathrm{C}$ rate discharge. However, under a $\mathrm{C} / 10$ discharge rate, $\mathrm{S}: \mathrm{CuS}$ hybrid electrodes exhibited lower sulfur utilization in the first discharge and faster capacity decay in later cycles than a pure sulfur electrode due to the dissolution of CuS. The CuS dissolution is found to be the result of strong interaction between the soluble low order polysulfide $\mathrm{Li}_{2} \mathrm{~S}_{3}$ and CuS. We identified the presence of conductive copper-containing sulfides at the cycled lithium anode surface, which may degrade the effectiveness of the passivation function of the solid-electrolyte-interphase (SEI) layer, accounting for the poor cycling performance of the S:CuS hybrid cells at low rate.
\end{abstract}

\footnotetext{
zE-Mail: hgan@bnl.gov
} 


\section{Introduction}

Li-S battery is amongst the most promising alternatives to Li-ion batteries, which are approaching their limits in energy density and power capability as determined by the metal oxide based cathode and graphite based anode materials. ${ }^{1}$ In spite of their high theoretical energy density $\left(2450 \mathrm{Wh} \mathrm{kg}^{-1}\right)$, Li-S battery systems under development still suffer from low power, low energy utilization and low efficiency due to factors such as the insulating nature of both sulfur and lithium sulfide, active material dissolution and the wells known shuttling effect. ${ }^{2}$ Successful remedies to these issues generally involved the use of novel sulfurs carbon cathode architectures. ${ }^{3 \mathrm{~s}} 5$ Meanwhile, transition metal sulfides have also been studied intensively as electrode materials in Li batteries in parallel to sulfur, because they serve as a good compromise between the high energy density of sulfur and better electronic conductivity in combination with a lower solubility of a metal based compound. ${ }^{6 s}{ }^{8}$ Since their electronic conductivities are close to or even exceeding that of graphite $\left(1000 \mathrm{~S} \mathrm{~cm}^{\mathrm{s}}{ }^{1}\right)$, these transition metal sulfides can work as conductive fillers for sulfur electrodes as good as carbon black and further contribute additional capacity to the existing Lis S battery to partially balance its fast capacity decay. ${ }^{9,10}$

CUS is a promising cathode material for lithium ion batteries due to its high theoretical capacity (560 mAh $\mathrm{g}^{\mathrm{s}}$ ) and flat discharge curve. ${ }^{11}{ }^{12}$ Specific discharge capacities up to $506 \mathrm{mAh} \mathrm{g}^{\mathrm{s}}$ have been demonstrated at the first cycle with good capacity retention over hundreds of cycles, ${ }^{13,14}$ which is on the highs end of capacity in the full spectrum of different transition metal sulfides. It should be noted that many transition metal sulfides, ${ }^{9,}{ }^{16}$ including $\mathrm{CuS}^{6}{ }^{6}$ can react with lithium metal at voltages in the vicinity of the working voltage $(\sim 2.1 \mathrm{~V})$ of Lis $\mathrm{S}$ battery, providing the opportunity for additional capacity. Furthermore, CuS is a good electronic conductor $\left(870 \mathrm{~S} \mathrm{~cm}^{\mathrm{s}}{ }^{1}\right){ }^{6}$ All above properties of CuS make it a promising candidate as a capacitys contributing conductive filler for the sulfur electrode. Recently, it has been reported that introduction of Cu nanos crystals into sulfur electrode actually helps to enhance the 
cyclability of Lis $\mathrm{S}$ battery by conversion of sulfur to more electrochemically stable CuS, ${ }^{10}$ which demonstrated the potential benefit of CuS inclusion for capacity retention of sulfur electrode.

The sulfur cell reaction involves a multistep chemical transformation with various electrolyte soluble polysulfide intermediates, which may enhance the interactions between the intermediates from sulfur and transition metal sulfides. Therefore the behavior of these two components in a hybrid electrode design using transition metal sulfide as capacity-contributing conductive additive could be completely different from the cases when they behaved individually. Up to now, the interactions between transition metal sulfide and sulfur along with related sulfur based species in a lithium-sulfur system are still not well understood.

In this work, we have investigated for the first time the electrochemical behaviors of coin cells constructed with the Sulfur-CuS hybrid electrode. The change in rate capability and utilization of sulfur with the amount of CuS additive is determined through a series of electrochemical tests. Using scanning electron microscopy (SEM) and transmission electron microscopy (TEM), we identify the presence and composition of a precipitated phase on the surface of Li metal anode, enabling elucidation of the mechanism of Sulfur-CuS interaction in these hybrid electrode cells. This study will help directing the development of novel conductive additives in Li-S battery and add to the understanding of the behavior of transition metal sulfides in their lithiation and delithiation processes.

\section{Experimental section}

Electrodes in this study were prepared with conventional methods by mixing active materials with carbon black (TIMCAL) and PVDF (Alfa Aesar) in 1s Methyls 2s pyrrolidone (Alfa Aesar) into a homogeneous slurry, which was casted onto an aluminum foil using a doctor blade. The electrode was dried in a fume hood under continuous dry air flow (dew point $<s \quad 40{ }^{\circ} \mathrm{C}$ ) for 24 hours before it was transferred into an oven to be heated at $50{ }^{\circ} \mathrm{C}$ for another 24 hours to eliminate residue solvent and moisture. To study the 
interaction of sulfur and CuS, 5 different active material compositions were employed by mixing sulfur (Alfa Aesar) and CuS (Alfa Aesar) in different molar ratios (Sulfur:CuS = 100:0, 90:10, 50:50, 33:67 and $0: 100$ ). CuS and $\mathrm{Cu}_{2} \mathrm{~S}$ (Alfa Aesar) slurries were also prepared with the above protocol and coated on one side of a separator (Celgard 2325) to make isolated interlayers for a different cell design, which will be introduced in the discussion session, to study interaction between polysulfides and CuS. 2032 coin cells were assembled by using electrodes prepared above as working electrode and a lithium disk as both counter and reference electrode, with 2 layers of Celgard (2325) separator in between to prevent shorting. Electrolyte used for testing of the Li-ion type cells was lithium hexafluorophosphate ( $\left.\mathrm{LiPF}_{6}\right)(1 \mathrm{M})$ in ethylene carbonate/dimethyl carbonate (EC/DMC) (volume ratio 50:50), and electrolyte used for testing of the Li-S cells and S:CuS hybrid electrode cells was Lithium bis(trifluoromethane)sulfonimide (LITFSI) (1M) dissolved in 1,2-dimethoxyethane (DME) and 1,3-dioxolane (DOL) (1:1 ratio, by volume) obtained from BASF. For the Li-S type cells, $1 \mathrm{wt} \%$ of $\mathrm{LiNO}_{3}$ was dissolved into the electrolyte before use to help the lithium electrode passivation and alleviate shuttling of polysulfide species. Electrochemical measurements were conducted at room temperature using a Maccor Cycle life tester or a Bio-logic (VMP3) battery cycler. Cells were cycled galvanostatically at $0.1 \mathrm{C}$ between $1.0 \mathrm{~V}$ and $3.0 \mathrm{~V}$ (for Li-ion type cells) or $1.8 \mathrm{~V}$ and $3.0 \mathrm{~V}$ (or $2.6 \mathrm{~V}$, for Li-S type cells) versus lithium. For rate tests, cells with pristine sulfur and S:CuS hybrid electrodes were discharged at $1 \mathrm{C}$ to $1.8 \mathrm{~V}$.

After the testing, lithium electrodes were recovered from the Li-S type coin cells and cleaned with DOL. Characterization of the reaction products on the surface of lithium will help to understand the interaction mechanism between sulfur and CuS, as it will be shown in the results. Morphology and composition of the lithium surface species were examined by scanning electron microscopy/energydispersive X-ray spectroscopy (SEM/EDS, JEOL 7600F). High-resolution TEM imaging and electron diffraction was also performed on the surface species in a TEM (JEOL $2100 \mathrm{~F}$ ). 
To measure the solubility of CuS in polysulfide solutions, $\mathrm{Li}_{2} \mathrm{~S}_{8}$ and $\mathrm{Li}_{2} \mathrm{~S}_{4}$ solutions in electrolyte were prepared. $70 \mathrm{mM} \mathrm{Li} \mathrm{S}_{8}\left(\mathrm{Li}_{2} \mathrm{~S}_{4}\right)$ solution is prepared by mixing $32.6 \mathrm{mg} \mathrm{Li} \mathrm{L}_{2}$ and 159.3 (68.2) $\mathrm{mg}$ sulfur in 20 $\mathrm{ml}$ electrolyte at $55{ }^{\circ} \mathrm{C}$ under magnetic stirring for 48 hours under Argon. After this preparation, $10 \mathrm{mg}$ of CuS was added to the polysulfide solutions and mixed at room temperature for one week. A Thermo Scientific ICAP 6000 was used for inductively coupled plasma-optical emission spectroscopy measurements. Five copper calibration standards were prepared $(0.1,0.5,1.0,5.0,10.0 \mathrm{ppm} \mathrm{Cu})$, and the resulting linear calibration curve was used $(r>0.99998)$ with $\lambda=324.7 \mathrm{~nm}$.

\section{Results and Discussion}

In order to determine an appropriate discharge condition for the hybrid cells which would maximize the usable capacity of the CuS additive, CuS cathodes were cycled at a $0.1 \mathrm{C}$ rate in two different voltage ranges: 3.0 V-1.8 V and 3.0 V-1.0 V vs. Li/Li', as shown in Figure 1. In the range of 3.0 V-1.8 V, a single voltage plateau was observed at $2.1 \mathrm{~V}$ with $245 \mathrm{mAh} \mathrm{g}^{-1}$ delivered on cycle 1, with $191 \mathrm{mAh} \mathrm{g}^{-1}$ remaining on cycle 10 and $109 \mathrm{mAh} \mathrm{g}^{-1}$ remaining on cycle 50, as shown in Figure 1a. In comparison, in the range of 3.0 V-1.0 V, two voltage plateaus were observed, at $2.1 \mathrm{~V}$ and $1.7 \mathrm{~V}$, with $434 \mathrm{mAh} \mathrm{g}^{-1}$ delivered capacity on cycle 1 , as shown in Figure $1 \mathrm{~b}$. However, the capacity degraded rapidly and there was only $7 \mathrm{mAh}^{-1}$ delivered on cycle 10. The capacity versus cycle number data is summarized in Figure 1c.

The observed voltage profiles agreed well with the previous reports on the cycling behavior of CuS. ${ }^{13,15}$ It has been reported that the higher voltage plateau at $2.1 \mathrm{~V}$ corresponds to the lithium insertion into CuS with the initial formation of $\mathrm{Li}_{\mathrm{x}} \mathrm{CuS}$ (equations 1 and 2 ), while the lower voltage plateau at $1.7 \mathrm{~V}$ is related to the conversion reaction to the composite of $\mathrm{Li}_{2} \mathrm{~S}$ and $\mathrm{Cu}$ (equation 3). ${ }^{15}$ In the second voltage plateau region, factors such as volume change during cycling, electrolyte decomposition and loss of active material due to the solubility of sulfide species contribute to the capacity degradation upon repeated cycling. ${ }^{6}$ Thus the $3.0 \mathrm{~V}-1.8 \mathrm{~V}$ window was identified to be the most appropriate window to 
maximize the usable capacity for the CuS additive, which is consistent with the cycling voltage window normally used for Li-S cell testing.

$$
\begin{aligned}
& \mathrm{CuS}+\mathrm{xLi}^{+}+\mathrm{xe}^{-} \rightarrow \mathrm{Li}_{x} \mathrm{CuS} \\
& 1.96 \mathrm{Li}_{x} \mathrm{CuS}+(2-1.96 \mathrm{x}) \mathrm{Li}^{+}+(2-1.96 \mathrm{x}) \mathrm{e}^{-} \rightarrow \mathrm{Cu}_{1.96} \mathrm{~S}+\mathrm{Li}_{2} \mathrm{~S} \\
& \mathrm{Cu}_{1.96} \mathrm{~S}+2 \mathrm{Li}^{+}+2 \mathrm{e}^{-} \rightarrow \mathrm{Li}_{2} \mathrm{~S}+1.96 \mathrm{Cu}
\end{aligned}
$$

CuS is hypothesized to be good capacity-contributing conductive additive for sulfur batteries. To determine the CuS impact on sulfur cell power capability, coin cells made from sulfur electrodes and hybrid electrodes were discharged under a $1 \mathrm{C}$ rate to $1.8 \mathrm{~V}$, where $15 \mathrm{wt} \%$ conductive carbon additive in the control cells (Sulfur:Carbon:PVDF $=45: 45: 10$ weight ratio) was replaced by the CuS additive in the hybrid cells (Sulfur:CuS:Carbon:PVDF $=45: 15: 30: 10$ weight ratio; Sulfur:CuS $=90: 10$ molar ratio). The $1^{\text {st }}$ discharge voltage profiles are shown in Figure 2. Cells with hybrid electrodes delivered $\sim 900 \mathrm{mAh} \mathrm{g}^{-1}$ based on sulfur weight, while the control cells without CuS additive delivered only 430-627 mAh g-1. Since CuS only contributes $5.3 \%$ of the total cell theoretical capacity in the hybrid electrode, this difference in delivered capacity is mainly contributed by the CuS induced power enhancement of the sulfur electrode under high rate discharge. More importantly, the hybrid electrode cells maintained the typical sulfur cell discharge voltage profile with two clearly distinguishable voltage plateaus at $2.3 \mathrm{~V}$ and $2.1 \mathrm{~V}$, while the sulfur control cells showed only the sloped voltage profile with severe voltage depression. This proof of concept test supports the hypothesis that the CuS may improve the power capability of the sulfur electrode when used as conductive additive in place of carbon black.

To understand the interaction between sulfur and CuS in the hybrid electrode and in comparison with sulfur control cells, lower rate cycling tests were executed. The impact of CuS additive on sulfur cell cycling was examined by adjusting the S:CuS ratio under $\mathrm{C} / 10$ cycling rate. The first discharge and 
charge curves of lithium cells with pure sulfur, CuS and Sulfur-CuS hybrid electrodes with various molar ratios are shown in Figure 3a. Except for the CuS cell, capacity in Figure 3 is normalized with respect to the mass of sulfur in the electrodes in order to make the part contributed by CuS additive clearer. CuS delivered a relatively reversible gravimetric capacity of $225 \mathrm{mAh} \mathrm{g}^{\mathrm{s}}{ }^{1}$ in the $1.8 \mathrm{Vs} 3.0 \mathrm{~V}$ voltage range, which corresponds to $80 \%$ theoretical capacity for the conversion of CuS into $\left[\mathrm{Li}_{2} \mathrm{~S}+\mathrm{Cu}_{2} \mathrm{~S}\right]^{15}$ by lithiation at $2.1 \mathrm{~V}$ versus lithium electrode. Intriguingly, in all cases with CuS additive, the hybrid electrode delivers less capacity than pure sulfur electrode in the first discharge at $C / 10$, in contrary to the observation under $1 \mathrm{C}$ rate discharge (Figure 2). The sulfur electrode itself delivers $880 \mathrm{mAhg}{ }^{\mathrm{s}}{ }^{1}$, which amounts to a utilization of $\sim 53 \%$ of sulfur, while all hybrid electrodes deliver less than $51 \%$ of the sulfur theoretical capacity, including the capacity contribution from CuS additive. In addition, even though electrolyte with $1 \mathrm{wt} \%$ of $\mathrm{LiNO}_{3}$ was used in all cases here, all hybrid electrodes demonstrate poor Coulombic efficiency by undergoing long charging sequences after the first discharge. The higher the CuS content in the hybrid electrode, the more severe the shuttling effect, leading to lower Coulombic efficiency. In the meantime, the pure sulfur electrode is consistent with prior literature by showing almost $100 \%$ Coulombic efficiency. The CuS electrode delivers $80 \%$ of its theoretical capacity above 1.8 $V$ and a $100 \%$ Coulombic efficiency in the first cycle. The cycling performance of different electrodes is shown in Figure 3b. Discharge capacities of hybrid electrodes all fall below $250 \mathrm{mAh} \mathrm{g}^{\mathrm{s}}{ }^{1}$ within four cycles (> 70\% capacity fade vs. $1^{\text {st }}$ cycle). In contrast, the pure sulfur electrode still maintains $450 \mathrm{mAh} \mathrm{g}^{\mathrm{s}}{ }^{1}$ after 20 cycles while the pure CuS electrode maintains $40 \%$ of its $1^{\text {st }}$ cycle capacity.

Assuming CuS only serves as a conductive additive and extra capacity reservoir to sulfur electrode, we expected that the hybrid electrodes with CuS additive deliver more capacity ( $\mathrm{mAh} \mathrm{g}^{-1}$ ) than the pure sulfur electrode using sulfur mass only to normalize the specific capacity. However, the hybrid electrodes unanimously delivered less capacity than pure sulfur electrodes for the $1^{\text {st }}$ discharge and also showed much faster capacity fade in later cycles. This clearly indicates that there is strong interaction 
between sulfur and CuS. The much longer 1st charging process shown by the hybrid electrodes suggests that the shuttling effect is more severe when CuS is added to the sulfur electrode. It is possible that cells with hybrid electrodes may fail to build up a stable passivation layer on lithium anode during the first discharge even in the presence of $\mathrm{LiNO}_{3}$. This non-passivated lithium surface would then be more reactive towards the reduction of high-order polysulfide into low-order ones, magnifying the shuttling effect in the $1^{\text {st }}$ charge process.

To understand the interaction mechanism, the surface of lithium anodes in cells with hybrid electrodes was investigated after cycling. It is observed that the surface of lithium anode in a cell with S:CuS=50:50 hybrid electrode covered with black deposit darker in appearance than that from either the sulfur cell or the CuS cell (Figure 4a). The black area coincides in shape with the $1.27 \mathrm{~cm}^{2}$ circular cathode used in this work. X-ray energy dispersive spectroscopy attached with SEM is performed over lithium foils recovered from cells with sulfur cathode, CuS cathode and S:CUS=50:50 hybrid cathodes after 20 cycles (Figure 4b). In addition to signals from O, C, F and S observed from cycled lithium foil in Li-S cell, the copper signature was revealed at $0.93 \mathrm{keV}(\mathrm{L} \alpha), 8.04 \mathrm{keV}(\mathrm{K} \alpha)$ and $8.90 \mathrm{keV}(\mathrm{K} \beta)$ in the other two cell types. SEM images of the surface of the three lithium foils are shown in the insets in Figure 4a. The anode surfaces from sulfur and CuS cathode cells are much smoother and less mossy than those from hybrid electrode cells. Sulfur and $\mathrm{Cu}$ mapping of the lithium anode surfaces (Figure 4c-e) showed homogeneous distribution. These observations indicate that both CuS and S:CuS=50:50 electrodes experienced CuS dissolution during cycling, which generates $\mathrm{Cu}$ cation in the electrolyte that is later deposited onto the lithium foil in parallel with sulfide and polysulfides. However, the darker lithium surface of hybrid electrode cell in Figure $4 \mathrm{a}$ and its more porous anode surface layer shown in figure $4 \mathrm{c}$ suggest that the deposition may be more significant in the hybrid electrode case. Sulfur in the hybrid electrode may interact with CUS and enhances its dissolution as to change the microstructure of the SEI layer on lithium. Figure $4 \mathrm{~g}$ is the selected area electron diffraction (SAED) pattern of one area of dark precipitation, a 
representative crystallite of which shown in Figure $4 \mathrm{f}$, obtained from a lithium surface belonging to a hybrid cathode cell. It is composed of concentric rings characteristic of polycrystalline materials. We identified the diffraction rings of $\mathrm{Li}_{2} \mathrm{~S}, \mathrm{Cu}_{2} \mathrm{~S}$ and $\mathrm{Cu}_{8} \mathrm{~S}_{5}$ in this region. Notably, similar to CuS, these reduced copper sulfides are also electronically conductive with conductivity as high as $2400 \mathrm{~S} \mathrm{~cm}^{-1}$. 6,17 The incorporation of metallically conductive copper sulfide species into the lithium sulfide based passivation layer on lithium may account for the intensive shuttling effect observed in the first charging and the fast capacity fade during the cell cycling for all hybrid electrode cells. Lithium surface passivation requires the build-up of an electronically insulating SEI layer. For the sulfur electrode cell, along with solvent reduction products, the non-conductive $\mathrm{Li}_{2} \mathrm{~S}$ deposit from polysulfide reduction are the major component of the SEl layer, which effectively slows down the kinetics of electron transfer across the SEI layer from lithium metal to the reducible species in the electrolyte at the interface, such as polysulfide. Under this situation, only the reduction of high-order polysulfides to soluble low-order polysulfides will occur before the diffusion of soluble low-order polysulfides away from the anode interface. Although this is not sufficient to completely prevent shuttling of polysulfide in the system, it avoids constant consumption of sulfur on the anode. However, when a significant amount of electronically conductive copper sulfide phasees is incorporated into the SEI layer, it will be much more difficult to develop a surface layer resistive enough to prevent the electron transfer across the SEI layer, resulting in the direct conversion of polysulfide to $\mathrm{Li}_{2} \mathrm{~S}$ deposition before the soluble intermediate will diffuse away from the lithium anode surface. The fast reduction of polysulfide to the insoluble $\mathrm{Li}_{2} \mathrm{~S}$ on the lithium anode surface consumes active sulfur in the cathode and causes severe capacity fade. In Figure $4 b$, it appears that the relative intensity of sulfur peak is much greater in the hybrid cell case than the other two cases, if oxygen peak is used as a reference, qualitatively supporting the hypothesis that more sulfur is lost from the system in the hybrid electrode cells. 
It is inferred that polysulfides formed during the reduction of sulfur interacted with CuS in the hybrid electrode and induced the dissolution, based on the fact that electrolyte soluble polysulfides are the only mobile species amongst all intermediates and products generated from sulfur and CuS. Polysulfide species are mostly generated within the sloped region above $2.1 \mathrm{~V}$ in the voltage-capacity profile of sulfur cell shown in Figure.3a. ${ }^{1}$ It has been established that $\mathrm{Li}_{2} \mathrm{~S}_{8}$ is the first stable intermediate generated by the reduction process of sulfur followed by a more complex step when $\mathrm{Li}_{2} \mathrm{~S}_{8}$ is converted into lowers order $\mathrm{Li}_{2} \mathrm{~S}_{\mathrm{n}}$ with $2<\mathrm{n}<8$, before all $\mathrm{Li}_{2} \mathrm{~S}_{\mathrm{n}}$ species begin to be reduced into $\mathrm{Li}_{2} \mathrm{~S}_{2}$ or $\mathrm{Li}_{2} \mathrm{~S}$ precipitate in the plateau region $\sim 2.0 \mathrm{Vs} 2.1 \mathrm{~V}$ of the discharge. To examine the hypothesis, solubility test of CuS in $\mathrm{Li}_{2} \mathrm{~S}_{n}$ solution is performed with the help of inductively coupled plasmas optical emission spectroscopy (ICPS OES). $\mathrm{Li}_{2} \mathrm{~S}_{8}$ and $\mathrm{Li}_{2} \mathrm{~S}_{4}$ solutions in electrolyte were made to study the interaction and dissolution. $\mathrm{Li}_{2} \mathrm{~S}_{4}$ was made because solutions of $\mathrm{Li}_{2} \mathrm{~S}_{\mathrm{n}}$ with $\mathrm{n}<4$ tends to undergo disproportionation and solute precipitation within a short period after solution preparation, probably due to the presence of reactive $\mathrm{S}_{3}{ }^{.5}$ radical. ${ }^{18}$ The ICPS OES test results of the 3 solutions are presented in Table 1. Surprisingly, copper was not detected in $\mathrm{Li}_{2} \mathrm{~S}_{4}$ or $\mathrm{Li}_{2} \mathrm{~S}_{8}$ solution that was stirred in the presence of CuS, while $6.9 \mathrm{ppm}$ of it was observed in CuS/electrolyte control sample.

The ICP-OES data in dicates that the interaction between $\mathrm{Li}_{2} \mathrm{~S}_{n}(n=8$ and 4$)$ and CuS in a nonelectrochemical environment does not induce CuS dissolution, but actually suppress the CuS dissolution in the electrolyte. To enhance the reliability of this measurement, a different experiment was performed in a coin cell. As mentioned earlier, $\mathrm{Li}_{2} \mathrm{~S}_{\mathrm{n}}$ mainly evolves at voltage above $2.1 \mathrm{~V} \mathrm{vs}$. $\mathrm{Li} / \mathrm{Li}^{+}$during the reduction of sulfur, while CuS does not start its initial lithiation process until $2.08 \mathrm{~V}$ as measured in Figure 3a. This suggests that if the lower cut-off voltage of the cycling of hybrid electrodes is set above $2.1 \mathrm{~V}, \mathrm{Li}_{2} \mathrm{~S}_{\mathrm{n}}$ formed will only come into contact with CuS. If polysulfides generated in this voltage range cause the CuS dissolution, then based on the hypothesis above the lithium anode surface should also be covered with $\mathrm{Cu}_{2} \mathrm{~S}$ containing dark deposition and presumably the capacity retention during the 
polysulfide cycling (2.1 V-2.6 V vs. $\left.\mathrm{Li} / \mathrm{Li}^{+}\right)$will be poor as the case of full-depth discharge already observed.

In the actual experiment, $2.15 \mathrm{~V}$ was chosen as the lower cut-off voltage for the discharge of the hybrid electrode to ensure no CUS was partially discharged, and based on the sulfur cell voltage-capacity profile in Figure $3 a$ this lower bound already covered $80 \%$ of the whole polysulfide discharge region. In the 20 cycles of capacity evolution shown in Figure $5 a$, it is seen that the $S: C u S=50: 50$ hybrid electrode only loses less than half of its initial capacity with $2.15 \mathrm{~V}$ discharge cut off limit while it is about a $75 \%$ loss within 4 cycles when $1.8 \mathrm{~V}$ cut-off is used (Figure $5 \mathrm{~b}$ ). A photo of the lithium anode recovered from the 2.15 V cut off cell is shown in Figure 5c, the surface of which only shows minor darkness, while the anode recovered from the $1.8 \mathrm{~V}$ cut off cell exhibited much darker color. These observations indicate that polysulfides generated above $2.15 \mathrm{~V}\left(\mathrm{Li}_{2} \mathrm{~S}_{4}, \mathrm{Li}_{2} \mathrm{~S}_{8}\right.$ etc. $)$ do not dissolve CuS, and this corroborates the results of the solubility test with ICP-OES.

The different appearance of the lithium anodes recovered from hybrid electrodes cycled with $2.15 \mathrm{~V}$ and $1.8 \mathrm{~V}$ lower cut-off voltage helps to identify the voltage onset range for dissolution to $2.15 \mathrm{~V}-1.8 \mathrm{~V}$. In this range $L_{2} S_{n}(n \geq 4)$ is further reduced to $\mathrm{Li}_{2} S_{n}(n<4), \mathrm{Li}_{2} \mathrm{~S}_{2}$ and $\mathrm{Li}_{2} S$, and CuS is lithiated to form $\mathrm{Cu}_{2} S$ and $\mathrm{Li}_{2} \mathrm{~S}$. Probably one or both of these two reactions must occur before the reactants responsible for deposit formation are present in the system. In order to identify the correct reactants, it was necessary to decouple the depth of discharge of sulfur and CuS in the cell, thus a new cell design with the incorporation of an isolated $\mathrm{CuS} / \mathrm{Cu}_{2} \mathrm{~S}$ coated interlayer is used to separate the two components, which is shown in Scheme 1. The discrete interlayer was isolated from both cathode and anode by additional separator layers.

Figure $5 \mathrm{~b}$ shows the cycle performance of sulfur cells in the $1.8 \mathrm{~V}-2.6 \mathrm{~V}$ voltage window with CuS and $\mathrm{Cu}_{2} \mathrm{~S}$ interlayers. It can be seen that the capacity fading for cells with $\mathrm{CuS} / \mathrm{Cu}_{2} \mathrm{~S}$ interlayers is just as fast 
as the case when a S:CuS=50:50 hybrid electrode is used. The lithium foils recovered from the interlayer design cells were also covered with significant black deposits (Figure $5 c$ ), behaving the same as the $\mathrm{S}:$ CuS=50:50 hybrid electrode cell. EDS analysis also detected significant amount of copper and sulfur signals in these dark regions, confirming that the black deposition is caused by $\mathrm{CuS}$ or $\mathrm{Cu}_{2} \mathrm{~S}$ dissolution and re-deposition on lithium. Combining this observation with information obtained with the $2.15 \mathrm{~V}-2.6$ $V$ cycling and solubility test of CuS in $\mathrm{Li}_{2} \mathrm{~S}_{n}(n=8$ and 4$)$ solutions, it is inferred that $\mathrm{Li}_{2} \mathrm{~S}_{n}$ has to be first reduced to $\mathrm{Li}_{2} \mathrm{~S}_{3}$ before the strong interaction starts. $\mathrm{Li}_{2} \mathrm{~S}_{2}$ and $\mathrm{Li}_{2} \mathrm{~S}$ should be taken out of the consideration since they mainly exist as insoluble solids in the discharged electrode. The reason for $\mathrm{Li}_{2} \mathrm{~S}_{3}$ to be more reactive towards $\mathrm{CuS}$ or $\mathrm{Cu}_{2} \mathrm{~S}$ probably lies in the fact that an $\mathrm{S}_{3}$ cluster is prone to form a radical in a polar electrolyte system. ${ }^{18}$

Additional study on $\mathrm{Cu}_{2} \mathrm{~S}$ interlayer cell was performed to elucidate the possible role of $\mathrm{Cu}_{2} \mathrm{~S}$ vs. CuS. A Li-S cell with an isolated $\mathrm{Cu}_{2} \mathrm{~S}$ interlayer was assembled and discharged to $1.8 \mathrm{~V}$. A similar degree of darkness as that shown in Figure $5 c$ was seen for the recovered anode after this discharge. After washing by DOL, the recovered $\mathrm{Cu}_{2} \mathrm{~S}$ interlayer was examined by XRD and SEM/EDS. The XRD diffraction peak of the pristine $\mathrm{Cu}_{2} \mathrm{~S}$ was not detectable from the recovered interlayer sample after just one discharge, while diffraction peak at $\sim 48^{\circ} 2 \Theta$ indicating possible formation of CuS (Figure S1). SEM images of the particles on the interlayer showed a decreased number density of particles after the discharge (Figure S2), and where it was also noted that the EDS signal ratio Cu:S decreased from 2.3 to 0.51 (Figure S3). Based on these observations, it is proposed that $\mathrm{Cu}_{2} \mathrm{~S}$ experienced both dissolution and conversion to CuS under the influence of polysulfide generated by the sulfur electrode discharge.

\section{Conclusion}

CuS has been studied as a capacity-contributing conductive additive to the sulfur electrode in a Li-S battery. CuS facilitates more efficient utilization of sulfur for the hybrid electrode in the initial discharge 
under $1 \mathrm{C}$ rate, whereas, under the $\mathrm{C} / 10$ discharge rate capacity enhancement is not observed. Upon repeated discharge/charge cycling, an adverse interaction between sulfur and CuS was indicated by higher fade for S:CuS composite electrodes. Observation and characterization of lithium anodes from S:CuS hybrid cells indicated Cu deposition, and a modified lithium anode surface morphology. Solubility tests of CuS in polysulfide solution and voltage-controlled charge/discharge tests indicated $\mathrm{Li}_{2} \mathrm{~S}_{3}$ is the species responsible for the dissolution of CuS. Although the exact chemistry for the dissolution of CuS by $\mathrm{Li}_{2} \mathrm{~S}_{3}$ is still not fully understood, which requires further work on characterization, however, we determined that CuS dissolution and the ensuing $\mathrm{Cu}_{2} \mathrm{~S}$ deposition on lithium surface enhance the active sulfur consumption on anode surface in the form of $\mathrm{Li}_{2} \mathrm{~S}$ deposition, contributing to the significant capacity fade of Li-S electrode.

Acknowledgement: This work is supported by the U.S. Department of Energy (DOE) Office of Energy Efficiency and Renewable Energy under the Advanced Battery Materials Research (BMR) program, Contract No. DE-SC0012704. Part of this work has been carried out at the Center for Functional Nanomaterials, Brookhaven National Laboratory, which is supported by the DOE, Office of Basic Energy Sciences, under contract DE-SC0012704. 


\section{References:}

1. P. G. Bruce, S. A. Freunberger, L. J. Hardwick, and J. M. Tarascon, Nat. Mater., 11, 19 (2012).

2. A. Manthiram, Y. Fu, S. H. Chung, C. Zu, and Y. S. Su, Chem. Rev., 114, 11751 (2014).

3. N. Jayaprakash, J. Shen, S.S. Moganty, A. Corona, L.A. Archer, Angew. Chem. Int. Ed., 123, 6026 (2011).

4. B. Zhang, X. Qin, G.R. Li, X.P. Gao, Energy Environ. Sci., 3, 1531(2010).

5. R. Elazari, G. Salitra, A. Garsuch, A. Panchenko, D. Aurbach, Adv. Mater., 23, 5641 (2011).

6. B. Jache, B. Mogwitz, F. Klein, and P. Adelhelm, J. Power Sources, 247, 703 (2014).

7. A. Débart, L. Dupont, R. Patrice, and J. M. Tarascon, Solid State Sci., 8, 640 (2006).

8. C. Villevieille, and P. Novák, J. Electrochem. Soc., 162, A284-A287 (2015).

9. Y. S. Su, and A. Manthiram, J. Power Sources, 270, 101 (2014).

10. S. Zheng, Y. Feng, Z. Li , Y. Zhu, Y. Xu, C. Luo, J. Yang, and C. Wang, Adv. Funct. Mater., 24, 4156, (2014).

11. G. Chen, Z. Wei, B. Jin, X. Zhong, H. Wang, W. Zhang, J. Liang, Q. Jiang, Appl. Surf. Sci., 277, 268, (2013).

12. M. Nagarathinam, K. Saravanan, W. L. Leong, P. Balaya, and J. J. Vittal, Cryst. Growth Des., 9, 4461, (2009).

13. Y. Wang, X. Zhang, P. Chen, H. Liao, S. Cheng, Electrochim. Acta., 80, 264 (2012).

14. Y. Du, Z. Yin, J. Zhu, X. Huang, X. J. Wu, Z. Zeng, Q. Yan \& H. Zhang, Nat. Commun., 3, 1177 (2012).

15. J.-S. Chung, H.-J. Sohn, J. Power Sources, 108, 226 (2002).

16. R. Fong, J. R. Dahn, and C. H. W. Jones, J. Electrochem. Soc., 136, 3206 (1989).

17. K. Okamoto and S. Kawai, Jpn. J. Appl. Phys., 12, 1130 (1973).

18. M. Cuisinier, C. Hart, M. Balasubramanian, A. Garsuch, and L. Nazar, Adv. Energy Mater., 1401801, (2015). 


\section{Captions:}

Table 1. Measured concentration of Copper in different solutions that have been mixed with CuS powder for one week.

Figure 1. (a) Voltage profile of Li/CuS electrochemical cell cycled between $3.0 \mathrm{~V}-1.8 \mathrm{~V}$; (b) Voltage profile of Li/CuS electrochemical cell cycled between 3.0 V-1.0 V; (c) Capacity versus cycle number of Li/CuS electrochemical cells cycled in two voltage ranges.

Figure 2. Discharge capacity and voltage profile of Sulfur cell and S:CuS hybrid cell under $1 \mathrm{C}$ rate.

Figure 3. (a) First cycle discharge-charge voltage profiles of Sulfur, $\mathrm{s}: \mathrm{CuS}=90: 10, \mathrm{~S}: \mathrm{CuS}=50: 50, \mathrm{~S}: \mathrm{CuS}=$ 33:67, and CuS electrodes. (b) Capacity retention of Sulfur, S:CuS $=90: 10, \mathrm{~S}: \mathrm{CuS}=50: 50, \mathrm{~S}: \mathrm{CuS}=33: 67$ and CuS electrodes in 20 cycles.

Figure 4. (a) Photos of lithium foils collected from Sulfur, CuS and S:CuS = 50:50 electrode cells after 20 cycles. Insets are corresponding SEM images, scale bar $=20 \mu \mathrm{m}$. (b) EDS spectrum of the lithium anodes shown in (a). (c) SEM image of lithium anode surface of S:CuS=50:50 cell. (d), (e) Sulfur and Copper signal mapping of (c). (f) TEM image of a cluster obtained from lithium anode surface of S:CuS=50:50 electrode cell, scale bar $=5 \mathrm{~nm}$. (g) SAED of the cluster shown in (f), scale bar $=2 \mathrm{~nm}^{-1}$.

Figure 5. (a) Capacity retention of two cells with $\mathrm{S}: \mathrm{CuS}=50: 50$ cathode with different cycling voltage ranges: $1.80 \mathrm{~V}-2.60 \mathrm{~V}$ and $2.15 \mathrm{~V}-2.60 \mathrm{~V}$. (b) Capacity retention of 2 sulfur cells with a $\mathrm{CuS}$ and a $\mathrm{Cu}_{2} \mathrm{~S}$ interlayer, respectively, S:CuS=50:50 electrode cell is shown as a reference. (c) Photos of surface of the lithium anodes obtained from cells listed in (a) and (b).

Scheme 1. Li-S cell with a CuS or $\mathrm{Cu}_{2} \mathrm{~S}$ interlayer coated on a Celgard separator that is isolated from both cathode and anode by additional separators. 
Table 1.

\begin{tabular}{|c|c|c|}
\hline Sample \# & Sample* & Cu Concentration (ppm) \\
\hline & & 0.0 \\
\hline 1 & Electrolyte for calibration & 6.9 \\
\hline 2 & Electrolyte + CuS & 0.0 \\
\hline 3 & $\mathrm{Li}_{2} \mathrm{~S}_{4}+$ Electrolyte + CuS & 0.0 \\
\hline 4 & $\mathrm{Li}_{2} \mathrm{~S}_{8}+$ Electrolyte + CuS & \\
\hline
\end{tabular}

*Electrolyte: 1.0M LiTFSI / DOL:DME = 1:1 v/v + 1wt\% $\mathrm{LiNO}_{3}$ 

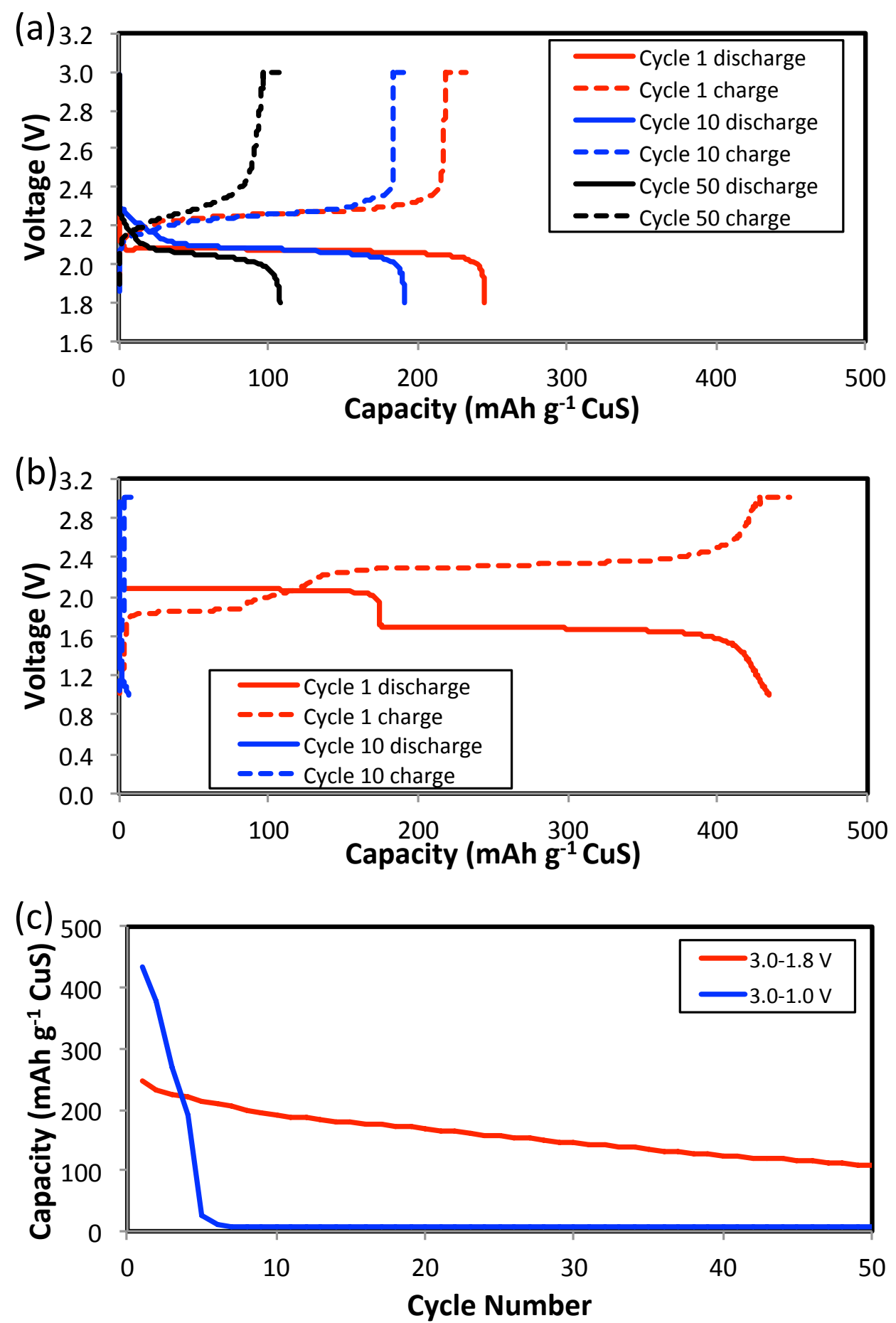

Figure 1. 


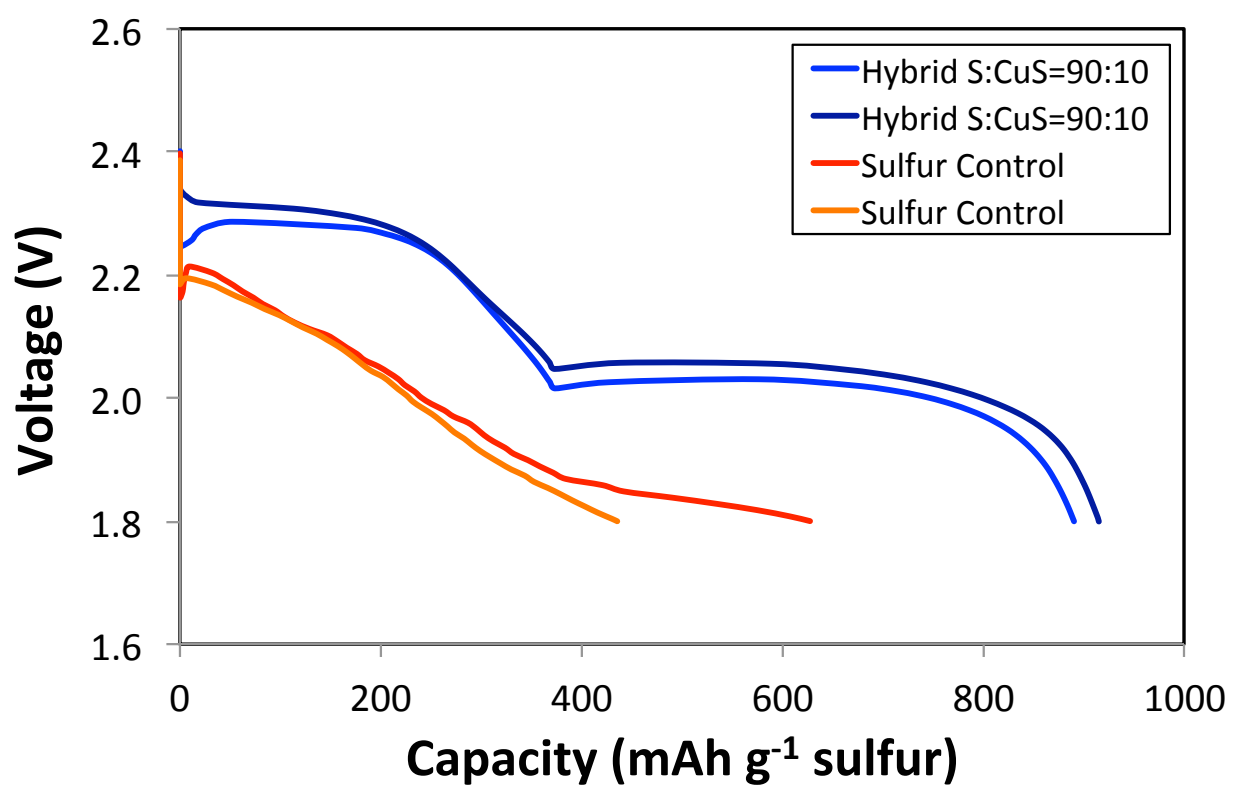

Figure 2. 

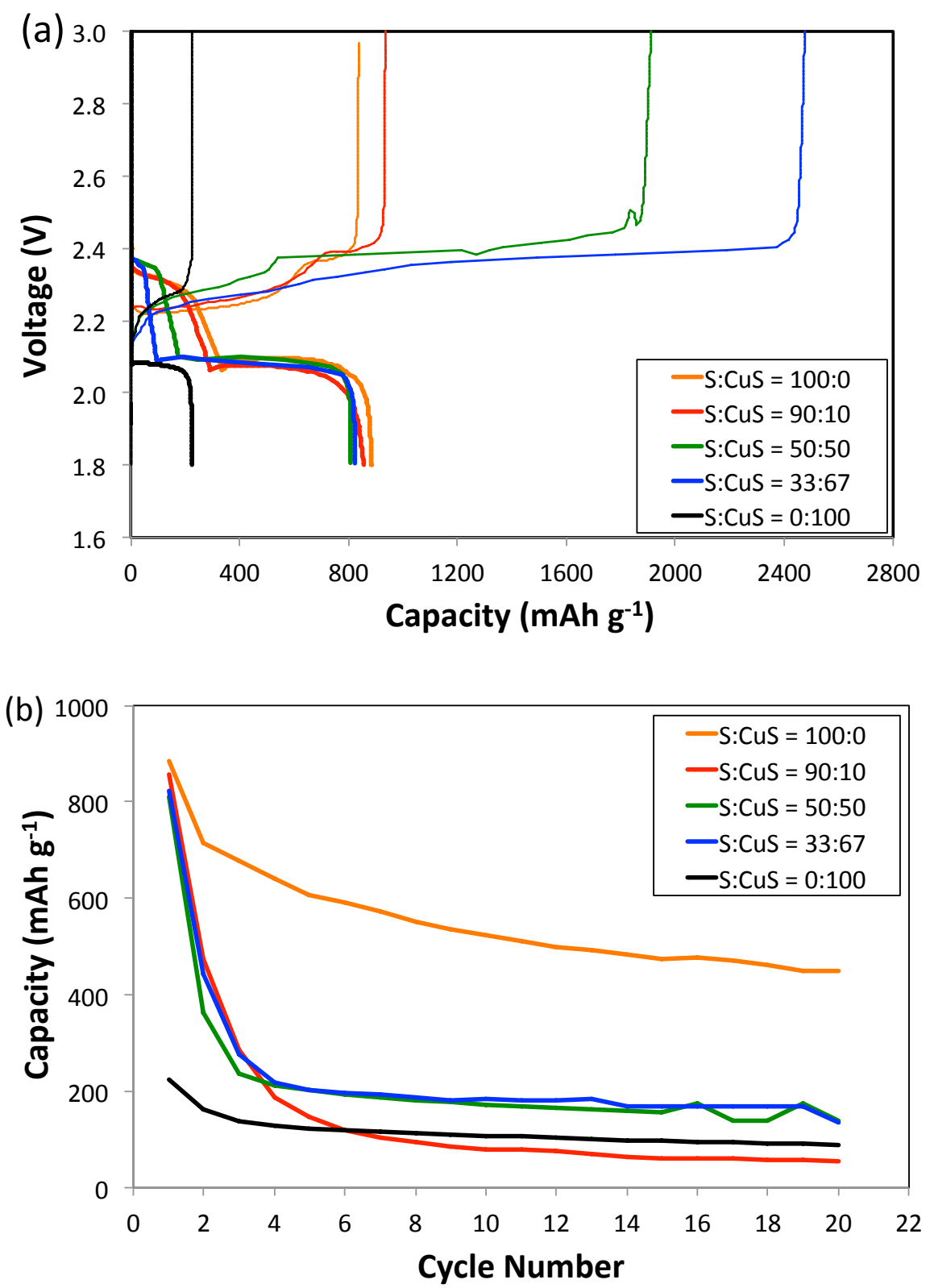

Figure 3 

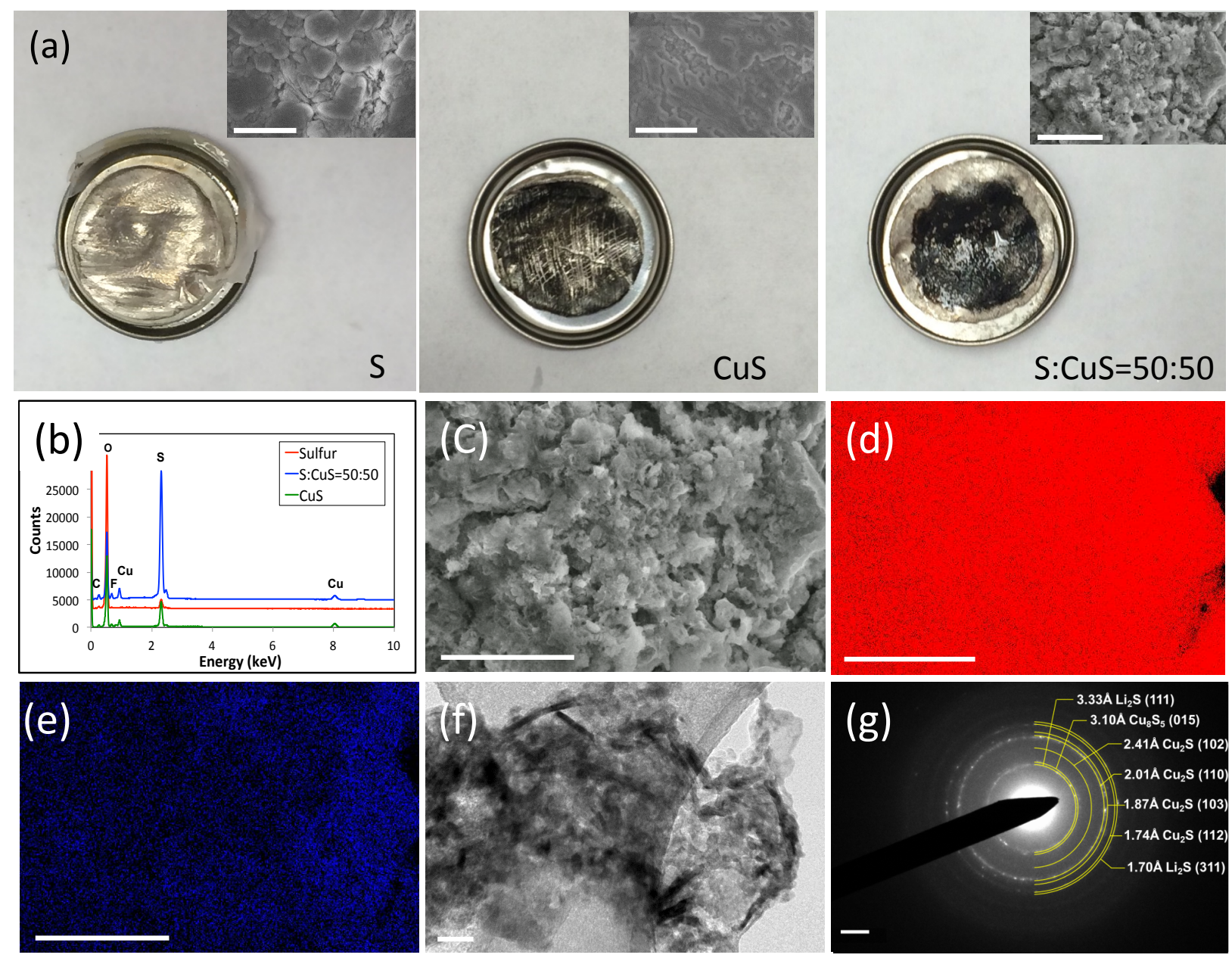

Figure 4 

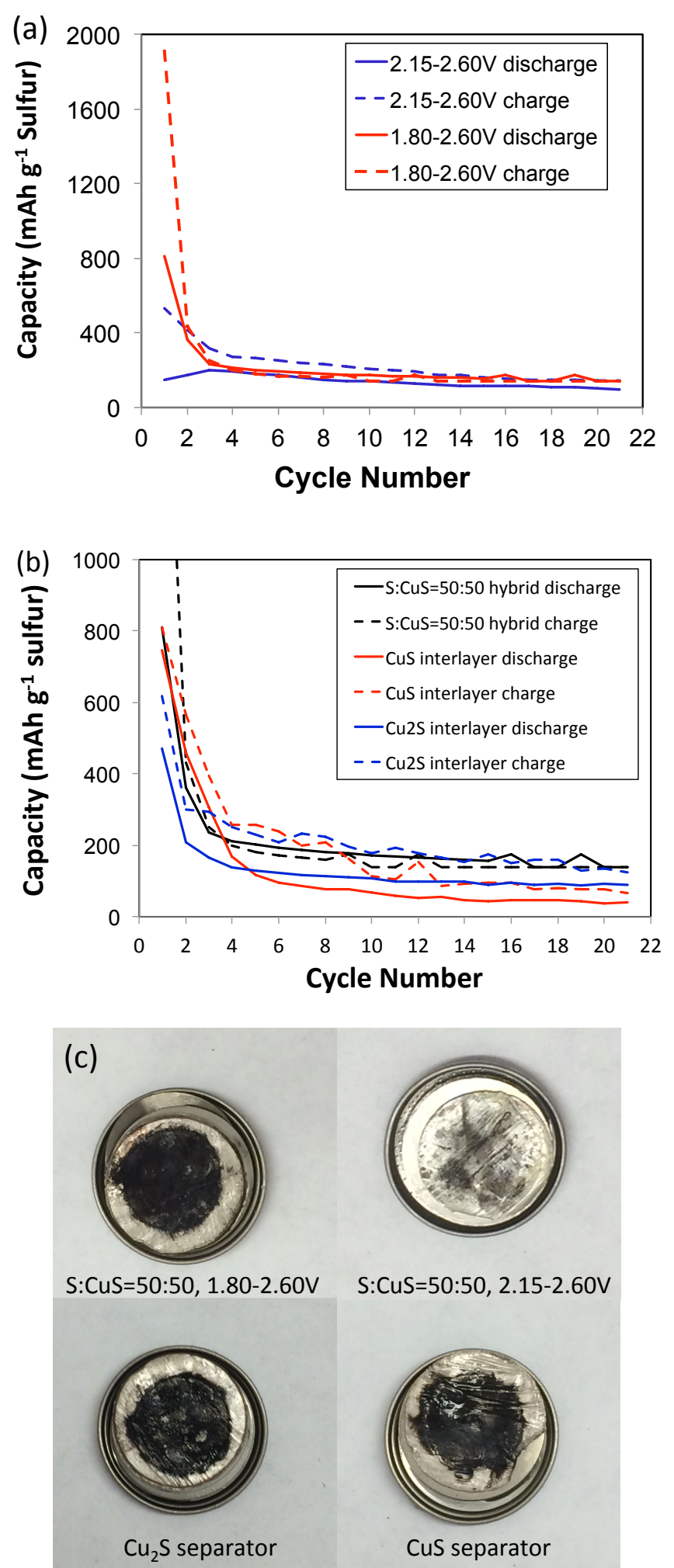

Figure 5 
Scheme 1

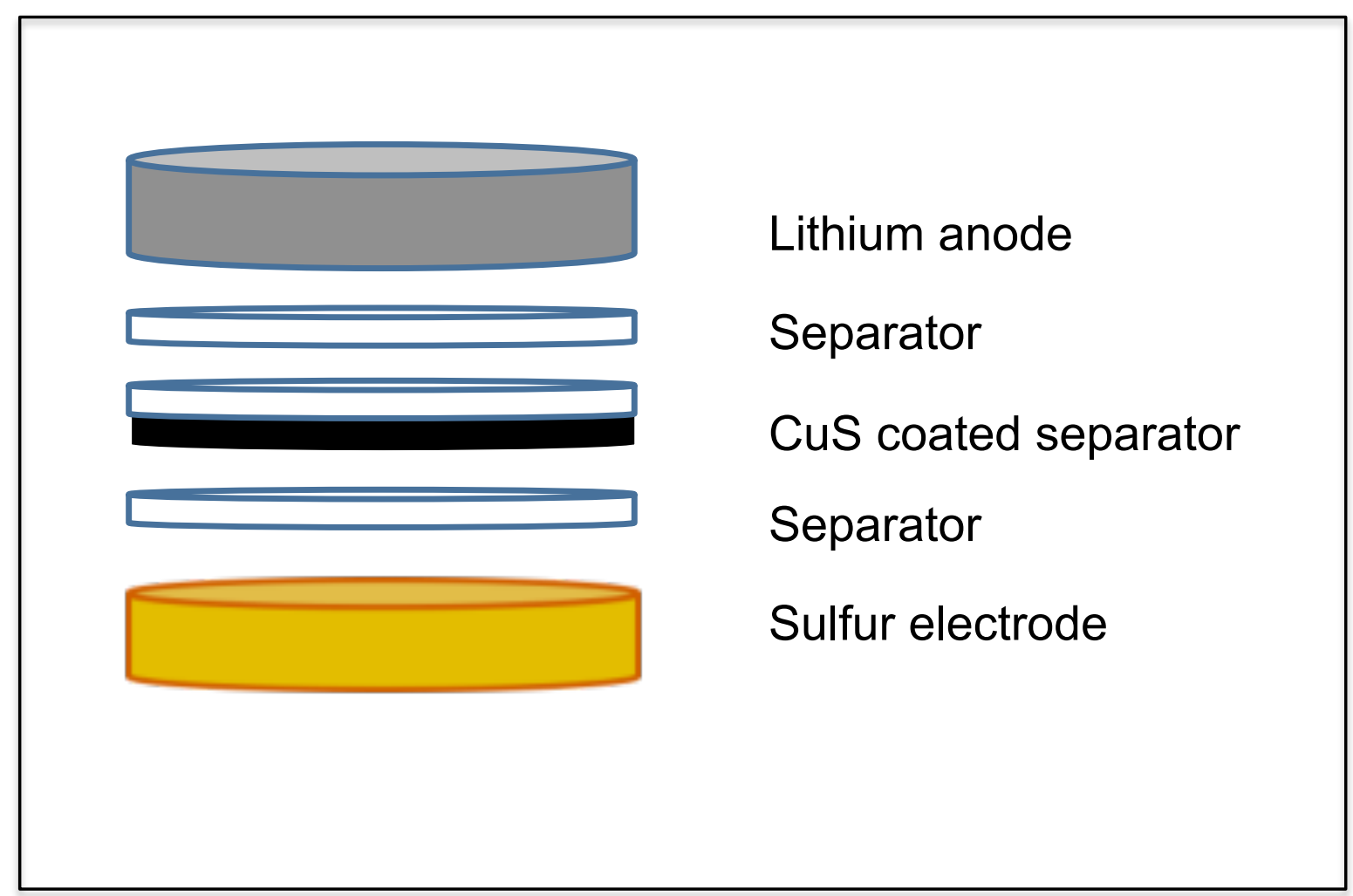

